\title{
EL MANEJO DEL DUELO EN CASOS DE HOMICIDIOS Y ASESINATOS EN PUERTO RICO
}

escrito por of

Nircia Del Rosario Meléndez

y Karisol Chévere Rivera

\section{Resumen}

Este artículo tiene como objetivo abrir un espacio para la reflexión y la discusión sobre un tema silenciado socialmente, a pesar de su cotidianidad: el análisis de los hallazgos de una entrevista a una familia puertorriqueña víctima del asesinato de un familiar. A partir de la Teoría de las Etapas de Kübler-Ross (1969) y la Teoría del Apego de Bowlby (1969, 1973, 1980) se analizan los hallazgos para esta familia. Además, se plantean las implicaciones para el Trabajo Social y profesiones afines, y la necesidad de promover y crear intervención y técnicas especializadas para atender el manejo del duelo, así como la educación respecto al tema. A partir de esta exposición, se espera que surjan más interrogantes y líneas de investigación que permitan profundizar en el tema, que es de gran impacto en la salud pública de Puerto Rico y Latinoamérica, así 
como aportar al quehacer del Trabajo Social Clínico en el país.

Palabras claves: manejo del duelo, pérdida repentina, homicidio/ asesinato, intervención

\section{Abstract}

The principal objective of this article is to open a space for the reflection and discussion on a socially muted theme despite its daily nature: bereavement. Bereavement deals with particularly the sudden loss of a person by a murder or homicide. It presents the results of an interview to a Puerto Rican family of which a member was the victim of a murder. The results of the interview are analyzed with the Kübler-Ross (1969) theory and the Attachment theory (Bowlby, 1969, 1973, 1980). In addition, it analyzes the implications for Social Work and related professions and the need to promote the creation of intervention and specialized techniques to address the issue, as well as providing education on the subject. As a result of this work, it is expected that additional questions and more research lines will arise on the topic, which is of great impact on public health in Puerto Rico and Latin America, contributing to the Clinical Social Work in the country.

Keywords: bereavement management, sudden loss, homicide/murder, intervention 


\section{Introducción}

Para muchas familias, la experiencia de la pérdida repentina de uno de sus integrantes es un proceso difícil de asimilar porque bajo estas circunstancias no se da un espacio para que los familiares se despidan del fallecido. Por lo sorpresivo del evento y la abrupta transición entre la vida y la muerte, muchas palabras quedan sin decir y surgen muchas interrogantes en los familiares, de las cuales pocas o ninguna tienen respuesta. Por tanto, subyace el pensamiento de que aún le faltaba por vivir. Esto implica una forma diferente de manejar la pérdida y el duelo, y nos remite a nuestra realidad social, donde diariamente las familias enfrentan la pérdida y el duelo repentino como resultado del dramático incremento de la criminalidad en el país.

El 2011 fue el año más violento enfrentado en la historia de Puerto Rico. Durante este año se registraron 1,164 asesinatos y homicidios, según las estadísticas de la Policía de Puerto Rico (Rivera, 2014). Este panorama prevaleció durante los siguientes años. En 2012, se cometieron 1,005 asesinatos y homicidios, y en el año 2013 se cerró la cifra con 883 (Rivera, 2014). En lo que va de este año, ya se han reportado 460 muertes de este tipo, de acuerdo con informes policíacos preliminares (Colón, 2014). En fin, el aumento en asesinatos en el país se ha convertido en la norma, y no en la excepción. Este panorama es congruente con el que vive el resto del mundo, donde en el 2012 se registró un total de 437 mil homicidios, y donde el continente americano se colocó con la mayor tasa de homicidios, representando un 36 por ciento (Oficina de las Naciones Unidas contra la droga y el delito, 2013).

Esta realidad nos pone de manifiesto la importancia de retomar el manejo del duelo por pérdida repentina, y comenzar a abrir espacios de reflexión en el cual se visibilice la discusión 
en torno a este tema. El manejo del duelo ha sido silenciado, estigmatizado y hecho invisible por nuestras sociedades capitalistas, las cuales han dictado la pauta de cómo sentir, pensar y actuar respecto a la muerte. De esta manera, se ha enmascarado e identificado como patológica la realidad de aquellos familiares que viven este proceso, sin que medie la alternativa y la posibilidad de asimilar el proceso con el apoyo y los recursos necesarios y adecuados a la particularidad de su historia o cultura. Muchos/as dolientes se valen de recursos religiosos para afrontar la pérdida. Sin embargo, surge la preocupación sobre las miles de personas que no poseen estas herramientas, recursos y alternativas para sobrellevar el proceso. Entonces, podrían desencadenarse patologías que, lastimosamente, inciden en la calidad de vida de los dolientes.

Este escrito es un intento por colocar en perspectiva la realidad de aquellos y aquellas que enfrentan el manejo del duelo por pérdida repentina, en específico, por homicidios y asesinatos, eventos que no son ajenos a nuestra realidad cotidiana, y quizás, por esa misma cotidianidad, los hemos naturalizado. Es por eso que como punto de partida se examinará el concepto de la muerte, para más adelante abordar el manejo del duelo por homicidio y asesinato desde la experiencia de una familia puertorriqueña. Esta es una realidad que trasciende hasta el contexto latinoamericano, reconfirmando la necesidad de abordar el tema, haciendo una mirada hacia el desarrollo y la implantación de intervenciones que atiendan la realidad específica de los dolientes.

Conjuntamente, se pretende analizar las implicaciones del tema para el Trabajo Social y otras profesiones afines, identificando específicamente las necesidades en el área de las intervenciones para atender el asunto en los familiares en duelo. Estas intervenciones deberían estar fundamentadas en 
investigaciones que profundicen en el tema. De esta manera, surgirán recursos para que el profesional del Trabajo Social Clínico pueda apoyar en estos procesos, discusión que se abordará para concluir este escrito.

\section{La muerte}

A pesar de la naturalidad de la muerte, para la sociedad actual es un tema vetado porque provoca incomodidad, amenaza, temor y negación. Papalia, Wendkos \& Duskin (2010) indican que "la muerte es un hecho biológico, pero también incluye aspectos sociales, culturales, históricos, religiosos, legales, psicológicos, del desarrollo, médicos y éticos, todos los cuales suelen estar estrechamente relacionados" (Papalia, Wendkos \& Duskin, 2010, p. 618). Añaden que la revolución de la mortalidad hace un contraste entre tiempos pasados, cuando era habitual la muerte en edades tempranas por causa de enfermedades de época, muertes neonatales hasta el primer año de vida y muertes de madres en labores de parto. Estas muertes repentinas se experimentaban con mayor sosiego (Kübler-Ross, 1969; Muñoz (1998) y Papalia et al, 2010).

En cambio hoy, los avances en la medicina, que incluyen nuevas alternativas de tratamiento para la cura y la prevención de enfermedades que antes fueron consideradas mortales; el acceso a servicios médicos y los cambios en los estilos de vida, que han acrecentado los años de existencia de las personas; y la educación han conducido a mayores esperanzas de sobrevivencia (Kübler-Ross, 1969 y Papalia et al, 2010). A partir del siglo 20, se desarrolla el concepto de la muerte desnaturalizada o muerte resignificada. Vázquez (2010) explica que en esta época surge un sujeto social ambicioso 
con expectativas de una vida prolongada, sin presencia de una reflexión sobre la vida o la muerte. "La muerte perdió sus significados sagrados y culturales, convirtiéndose en objeto intervenido por la ciencia, principalmente a través de la medicina" (Vázquez, 2010, p. 309). Sin duda, el dolor por la pérdida y la manera de llevar el duelo ha evolucionado con el tiempo.

Esta concepción de la muerte, como un elemento negativo, se acrecienta cuando sucede en etapas tempranas y medianas de la vida, transformando el sentimiento de la pérdida en uno más doloroso. Este planteamiento lleva a prestar atención a lo que sucede cuando la muerte es inesperada, debido a que el manejo del duelo por muerte repentina es un proceso que tiende a prolongarse por mayor tiempo en comparación a los demás; y que, a su vez, ese retraso en la recuperación de la pérdida puede desencadenar en un duelo complicado o patológico, desarrollando conductas no adaptativas que afectan a la persona en un nivel físico, emocional, mental, social y económico (Cabodevilla 2003, 2007; Gamo \& Pazos, 2009; Jacinto, de Barros \& Pelloso, 2008; Lindemann, 1944 en Fast, 2003; Meyers et al., 2009).

Como menciona Kübler-Ross (1969):

"Para nuestro inconsciente es inconcebible imaginar un final para nuestra vida aquí en la tierra, y si la vida nuestra tiene que terminar, el final siempre es atribuido a una intervención maligna del exterior causada por alguien más (...) Por tanto, la muerte en sí es asociada con un acto malo, un suceso aterrador, algo que en sí mismo exige venganza y castigo (Kübler-Ross, 1969, p.5; traducido por la investigadora)". 
De esta manera, la muerte se ha convertido en un concepto vetado cultural, social e individualmente. Desde el plano educativo, no se nos enseña a manifestar el duelo y a trabajarlo. Por lo tanto, al sujeto no le queda opción más que aguantar y ocultarlo. Los temores y la negación de la muerte han transformado el sentimiento de la pérdida en uno más doloroso, más aún si la muerte llega más temprano de lo esperado. Se hace inevitable sentir aflicción al perder, por causa de muerte, a un ser querido, aunque en la mente exista la conciencia de que la muerte forma parte del desarrollo humano. Se siente dolor e incomprensión, en muchas ocasiones, por un tiempo prolongado y/o indeterminado.

\section{Una mirada al manejo del duelo por homicidio/asesinato en Puerto Rico}

Respecto a la especificidad del tema de la pérdida repentina, existen limitados estudios y literatura al respecto. Algunos de los hallazgos, a nivel local, hacen referencia a los estudios y publicaciones de Muñoz (1998), Vázquez (2010, 2011), López (2013) y Alemán (2014). A partir de esta necesidad, se realizó una investigación del tema que profundizara en la realidad de las familias puertorriqueñas (Del Rosario-Meléndez, 2013), con el fin de aportar al quehacer del Trabajo Social Clínico y profesiones afines en el país. El propósito de este estudio fue explorar la experiencia, el proceso de asimilación y el manejo del duelo por homicidio/asesinato, accidente o muerte súbita, esta última entendiéndose que no hubo un diagnóstico previo ni enfermedad precedente al deceso, en seis participantes con una pérdida bajo una de estas tres circunstancias. 
A pesar de que la referida investigación abordó la muerte inesperada desde tres perspectivas, para propósitos de esta publicación se abundará solamente en la categoría de homicidio/ asesinato. El abordaje de este tipo de pérdida en particular parte del entendimiento de que la alta incidencia de homicidios y asesinatos en el país en los últimos años merece atención, sobre todo porque se ha promovido la naturalización de esta práctica de homicidio/ asesinato en la sociedad puertorriqueña. Hablamos de que miles de personas están experimentando anualmente un duelo sorpresivo, cargado por el arrebato de la vida sin sentido, sin tener el control y, en algunos casos, con un proceso judicial que prolonga la posibilidad de un cierre de ciclo. Esto ocurre, en muchas ocasiones, sin mediar un manejo adecuado de esta multiplicidad de emociones, pensamientos y pérdidas.

Para analizar el manejo del duelo por homicidio/ asesinato, se utilizaron como teorías bases de esta investigación la Teoría de las Etapas (Kübler-Ross, 1969) y la Teoría del Apego (Bowlby, 1969, 1973, 1980). En su teoría, Kübler-Ross (1969) propuso cinco etapas del duelo: rechazo, que es no aceptar la muerte; coraje, que entremezcla emociones de tristeza, hostilidad y culpa hacia otros; negociación, pedir a Dios que le regrese a la vida; depresión, en la que se altera la rutina; y aceptación, se reconoce la muerte y se continúa con el curso de la vida. La Teoría del Apego (Bowlby, 1969, 1973, 1980) "intenta describir y explicar la formación, desarrollo y pérdida de los vínculos afectivos desde el nacimiento hasta la muerte", (Bowlby, 2009, p.10) lo que permite explorar el manejo de este proceso de duelo por pérdida repentina y las diversas reacciones basados en los lazos afectivos creados entre los integrantes de la familia. En otras palabras, la intensidad del duelo depende del tipo de relación, sea distante, típica o estrecha, fomentada a través del tiempo entre el fallecido y el familiar. 
A partir de un enfoque cualitativo se analizó e interpretó cada una de las experiencias de los participantes, que incluyen el significado del duelo, los sentimientos y las emociones a partir de la muerte de su familiar. El alcance fue exploratorio y descriptivo, y se utilizó el diseño fenomenológico. Este diseño posibilitó capturar los sentimientos, las ideas, las emociones y las acciones de cada participante para explicar su proceso de pérdida repentina, identificado como el fenómeno. Una guía de entrevista semiestructurada permitió obtener información de la diada de duelo por pérdida repentina, en la categoría de homicidio/asesinato. Luego se realizó un análisis de contenido mediante un acuerdo entre jueces para categorizar las respuestas de los participantes. Los acuerdos de las categorizaciones fueron organizados y analizados utilizando el programa de análisis cualitativo $N$ Vivo.

Los participantes de la díada del proceso de asimilación y el manejo del duelo por homicidio/asesinato fueron la madre y el padrastro de un joven de 26 años, quien fue asesinado "a mansalva" en julio de 2013. Las entrevistas semi-estructuradas se llevaron a cabo de manera independiente. Es decir, la madre y el padrastro fueron entrevistados en tiempos distintos. Se recopilaron los relatos de ambos participantes y los diferentes matices de la pérdida repentina del hijo. De los resultados, llamó la atención la experimentación de las etapas del duelo (Kübler-Ross, 1969) y el concepto de apego (Bowlby, 1969, 1973, 1980). En adelante, presentamos los resultados de las categorías que tocaron ambos conceptos teóricos. 


\section{Etapas del duelo}

Las expresiones de la primera participante de esta díada de homicidio/asesinato evidencian un proceso de reflexión sobre esta muerte, que muy bien puede ser visto desde las etapas establecidas por Kübler-Ross. "Es difícil lo que uno pasa, lo que uno piensa, lo que uno analiza (...) Por qué me pasó a mí. Porque no hay un por qué, sencillamente pasó", expresó la madre del joven asesinado. En esta verbalización, hay una manifestación de los pensamientos, las emociones y los sentimientos que entremezclan la experimentación de dolor, coraje, cuestionamientos, angustia, hasta tratar de aceptar lo sucedido por parte de una madre cuyo hijo fue asesinado. Esta aceptación está enmarcada en la verbalización de "yo sé que él está bien y que las personas tienen que seguir viviendo", añadió la madre. No obstante, el reconocer la muerte y la continuidad de la vida, no impide que las personas recuerden sucesos, experiencias, ideas o emociones que constituyen una etapa antecesora. "Primero sentía dolor, matarlo con mis propias manos, beberme la sangre, destriparlo, etcétera, etcétera. Si en ese momento hubiese tenido la oportunidad, lo hubiese hecho feliz", manifestó la progenitora. Este es un ejemplo del coraje y frustración que aún experimenta la madre ante este suceso.

\section{Apego}

Para analizar el papel del apego en estos duelos, se presenta la verbalización del padrastro de la víctima, quien asumió el rol de padre del fallecido desde sus primeros años de vida. Este indicó que "mi hijo y yo éramos parecidos en carácter, en el temperamento, en decisiones, en todo lo que 
tenga que ver en lo emocional. Se me ha hecho un poco difícil el poder asimilar ese aspecto de no tener su presencia". La identificación de sus características, conductas o rasgos en los comportamientos de su hijastro permitió establecer lazos afectivos fuertes, así como un vínculo estrecho entre ellos. Por eso, anhela su regreso y busca la persona perdida. Ese reclamo para que su hijastro retorne a la vida llama la atención, debido a la connotación que transmite sobre una posible negación de la pérdida. Esta presunción se confirma cuando el participante indica que "yo siempre me quedo sentado en la misma silla esperando que me llame(...) Para mí, mi hijo está en un viaje", expresó el considerado por el fallecido como papá. La espera de la pronta llegada confirma su negación de despedirse del joven asesinado. Esta verbalización es consistente con la etapa de no asimilar la pérdida, por lo que la rechaza. Aquí se observa cómo el apego se entrelaza e influye en la experimentación de las etapas de duelo.

Ambos testimonios presentan un contraste entre miembros de una misma familia, quienes tienen en común la muerte, en este caso, la muerte de un hijo. En las verbalizaciones se presenta los diferentes niveles y maneras de manejar y experimentar el proceso de muerte inesperada. Comúnmente, se habla de un solo tipo de duelo en general. En este asunto es evidente y pertinente hablar de los duelos: el duelo personal y diametralmente diferente entre la mamá y el padrastro, y el duelo familiar. Esto confirma la pertinencia de trabajar específicamente el duelo por pérdida repentina por homicidio/ asesinato desde ambos enfoques, individual y familiar, más allá de los constructos sociales y personales.

Entre los resultados se destaca la reafirmación de una de las mayores críticas a la Teoría de las Etapas. Resulta que en estas etapas no hay jerarquía ni orden, sino se experimentan 
simultáneamente. Esto contrasta con el planteamiento inicial en el que se establecen las etapas de manera lineal, como si se tratase de cinco escalones que van siendo superados hasta completar el ciclo con la aceptación de la muerte. Por el contrario, una persona adelanta y retrocede en esta clase de espiral que son las etapas. Ese familiar puede aceptar la muerte, pero esto no lo limita a sentir por momentos coraje o cualquiera de las otras etapas, ya que puede experimentar más de una a la vez, tal y como lo demostraron los participantes de esta categoría.

En cuanto al apego, la intensidad del duelo depende del tipo de relación fomentado a través del tiempo entre el fallecido y familiar (distante, típico o estrecho). Mientras más estrecho es el apego, más marcadas las etapas del duelo que denotan negación o coraje, según demostrado por ambos participantes. Esto no limita la capacidad de estos, en el caso particular de mamá, de alcanzar la aceptación como parte del proceso.

\section{Implicaciones para el Trabajo Social}

Dentro de las implicaciones para el Trabajo Social y profesiones afines, el gran impacto en el sistema familiar y su entorno que genera el duelo por muerte repentina requiere especial atención. En las muertes por homicidio/asesinato se entremezcla el elemento inseguridad del entorno, temor por la vida y noción de injusticia. Se suma la incomprensión social del evento. Las personas en duelo entienden que su dolor se debe vivir a puerta cerrada. Culturalmente, se ha precisado un tiempo para sanar. Se ha creado la percepción que el duelo debe ser un proceso rápido y a escondidas. Estas acciones son injustas y obligan a la persona en duelo a tragarse sus emociones. "La 
actitud social ante los duelos, en nuestro medio, es de presión hacia su ocultación y aislamiento" (Cabodevilla, 2007, p. 165). Las heridas sin sanar afectan la estabilidad física, emocional y psicológica del deudo.

Los asesinatos están tan cerca de todos, el duelo por esta pérdida toca a muchos, pero la sociedad niega y oculta esta realidad. Se habla del duelo, pero no se profundiza. Si morir es un tabú, ser asesinado adquiere mayores dimensiones y repercusiones para los familiares sobrevivientes. Entran a escena elementos de culpabilidad, desconfianza, justicia a medias, estigmatización, entre otros. Cada una de estas situaciones juega con la estabilidad, salud mental y bienestar del familiar sobreviviente.

Este tema tiene repercusiones más allá de nuestro entorno. Aunque la investigación realizada tiene como enfoque la población puertorriqueña, el tema en discusión es aplicable a la realidad social en Latinoamérica. A manera de ejemplo, resalto el insumo recogido por parte del público que asistió a la presentación de parte de estos hallazgos en la V Congreso Regional de la Sociedad Interamericana de Psicología en la Universidad Centroamericana José Simeón Cañas (UCA) en San Salvador, El Salvador el verano pasado. Para contextualizar, en El Salvador se han librado varias batallas. Su historia está marcada por una guerra civil que duró 12 años (1980-1992) dejando sobre 70 mil muertos, ocho mil desaparecidos y muchos más con duelos por muerte repentina no trabajados, los cuales se ocultan detrás de una denominada cultura del silencio que atenta contra la memoria colectiva. Luego de este conflicto armado, comenzó la era de las pandillas, cuyos asesinatos, entre ellos y contra los ciudadanos, han sembrado el terror en las calles. La violencia política, la desigualdad y pobreza abonan al terreno. Al cierre de agosto de 2014, se 
habían registrado 2,533 asesinatos, superando los 2,491 informados al culminar el 2013, de acuerdo con la Policía Nacional Civil (Melara et al., 2014). Esto es un promedio de 10 asesinatos por día en un país con una población que supera los seis millones de habitantes.

A pesar de que esta sociedad vive tan de cerca la muerte repentina, en particular producto de los asesinatos, son pocas las técnicas disponibles para manejar estos casos, tal y como ocurre en Puerto Rico. Esta experiencia ante un público latinoamericano permitió contextualizar la realidad actual en Puerto Rico ante el incremento de la criminalidad, pero sobre todo abrió un espacio de reflexión que permitió, en primer lugar, la reapertura del pensamiento y discusión de un tema silenciado y, en segundo lugar, entender que de acuerdo con la cultura, el contexto y las situaciones y conflictos de cada país o región, el manejo de la pérdida repentina toma un nuevo giro, aunque con un desenlace parecido. Se reconfirmó la urgencia de atender con particularidad esta clase de duelos en cualquier parte del mundo. Por esto, se recomienda ampliar al resto del país esta línea de investigación y, posteriormente, extenderla hacia la cultura latinoamericana. 


\section{A modo de conclusión}

Entre las recomendaciones, se prioriza el desarrollo de técnicas de intervención especializadas e individualizadas para el duelo por pérdida repentina. En estas se debe considerar la comprensión de las etapas del duelo, tanto por parte del deudo como del profesional, amparados en el reconocimiento y expresión del dolor; la experimentación del duelo basada en la etapa de desarrollo del fallecido y el sobreviviente; y el tipo de vínculo afectivo establecido.

A nivel social, se recomienda fomentar, desde etapas del desarrollo tempranas, una educación basada en la preparación y aceptación de la muerte. Esta pedagogía de la muerte daría un nuevo giro a la concepción de la muerte desnaturalizada y crearía un nuevo abordaje de la muerte, alejado del tabú y la negación. Los profesionales de la conducta requieren adiestrarse en cuanto a las estrategias sobre el manejo de la muerte en sus diferentes manifestaciones, para así liderar la batalla de romper los esquemas en torno a la muerte y el duelo. Deberán, además, trabajar en conjunto el duelo individual y el familiar. La labor de reeducar sobre la muerte, incluyendo el manejo de la pérdida repentina, específicamente causada por los homicidios y asesinatos, es una tarea que solicita el compromiso, tanto de los profesionales, como de la sociedad y el propio individuo. Para sanar, el ser humano tiene derecho a comprender su pérdida y su dolor. 


\section{Referencias}

Alemán, Ada (2014, marzo). Los determinantes sociales de la salud y su impacto en el proceso de muerte y duelo. Ponencia presentada en el Simposio "Determinantes sociales de la salud: acciones desde la Psicología y prácticas afines" de la Asociación de Psicología de Puerto Rico, Universidad del Sagrado Corazón, San Juan, Puerto Rico.

Bowlby, John (1969). Attachment and loss. Attachment, vol.1. New York, Estados Unidos: Basic Books.

Bowlby, John (1973). Attachment and loss. Separation, Anxiety and anger, vol.2. New York, Estados Unidos: Basic Books.

Bowlby, John (1980). Attachment and loss. Loss, vol.3. New York, Estados Unidos: Basic Books.

Bowlby, John (1993). La pérdida afectiva. Barcelona, España: Editorial Paidós.

Bowlby, John (2009). El apego. El apego y la pérdida (volumen I). Buenos Aires, Argentina: Editorial Paidós.

Cabodevilla, losu (2007). Las pérdidas y sus duelos. Anales del sistema sanitario de Navarro, 30(3), 163-176. http://dx.doi. org/10.4321/S1137-66272007000600012

Colón, Javier (1 de septiembre de 2014). Violento el fin de semana. Primera Hora, p. 14-15.

Del Rosario-Meléndez, Nircia (2013). Manejo del duelo por pérdida repentina por homicidio/asesinato, accidente $y$ muerte súbita. Proyecto de investigación inédito. Universidad del Este, Carolina: Puerto Rico.

Diccionario Jurídico. Recuperado de www.lexjuridica.com

Fast, Jonathan (2003). After Columbine: how people mourn sudden death. Social Work, 48(4), 484- 
491. Recuperado de http://www.thefreelibrary.com / After+Columbine\%3A+how+people+mourn+sudden +death.-a0110727222

Gamo, Emilio; Pazos, Pilar (2009). El duelo y las etapas de la vida. Revista de la Asociación Española de Neuropsiquiatría. 29(2). 455-469. http://dx.doi.org/10.4321/S021157352009000200011

Jacinto, Ana; de Barros, María Dalva \& Pelloso, Sandra (2008). La muerte de un hijo joven en circunstancias violentas: comprendiendo la vivencia de la madre. Revista LatinoAmericana de Enfermagem, 16(3). Recuperado de www. eerp.usp.br/rlae

Kübler-Ross, Elizabeth (1969). On death and dying. Gran Bretaña: Tavistock Publications Limited.

Melara, Gabriela; López, Marta; Santos, Jessel; \& Ávalos, Jessica (1 de septiembre de 2013). Asesinatos hasta agosto 2014 superan los de todo 2013. La Prensa Gráfica. Recuperado de: http://www.laprensagrafica. com/2014/09/01/asesinatos-hasta-agosto-2014-superanlos-de-todo-2013

Meyers, Karen; Golden, Robert; Peterson, Fred \& General Editors (2009). The truth about death and dying. [Segunda edición]. Recuperado de la Base de Datos Infobase Ebooks Fulltext.

Muñoz, María (1998). La muerte toda una vida para enfrentarla. Universidad del Este, Carolina Puerto Rico: Editorial Talleres.

Papalia, Diane; Wendkos, Sally \& Duskin, Ruth (2010) Manejo de la muerte y el duelo. En Desarrollo Humano [11ma. Edición] (pp. 616-638). México, D.F.: The McGraw-Hill Companies, Inc. 
Rivera, Miguel (13 de enero de 2014). Ciencias Forenses confirma 917 homicidios. El Vocero, p. 16.

Oficina de Naciones Unidas contra la droga y el delito (2013). Estudio mundial sobre el homicidio. Recuperado de: https://www.unodc.org/documents/gsh/pdfs/GLOBAL_ HOMICIDE_Report_ExSum_spanish.pdf

Vázquez, Angie (2010). Para comprender la muerte: Análisis del discurso social, ritos, representaciones y significados. San Juan, Puerto Rico: Bibliográficas.

Vázquez, Angie (2011). Duelo que me duele: abordaje psicoterapéutico al proceso de duelo Para comprender la muerte: Análisis del discurso social, ritos, representaciones y significados. San Juan, Puerto Rico: Bibliográficas. 